\title{
Developing Detectors for Scintillation Light in Liquid Argon for DUNE
}

\author{
Bruce Howard ${ }^{* \dagger \ddagger}$ \\ Indiana University \\ E-mail: bruhowar@indiana.edu
}

The Deep Underground Neutrino experiment will conduct a broad program of physics research by studying a beam of neutrinos from Fermilab, atmospheric neutrinos, neutrinos from potential supernovae, and potential nucleon decay events. In pursuit of these studies, the experiment will deploy four 10kt fiducial mass liquid argon time projection chambers underground in Lead, South Dakota. Liquid argon time projection chambers allow high-resolution tracking and energy measurements. A precise timing signal is needed to provide the necessary time stamp to localize events in the drift direction. As liquid argon is a natural scintillator, a photon detection system will be deployed to provide such a signal, especially for non-beam events. In the baseline design for the single-phase time projection chamber, the detectors are contained within the anode plane assemblies. The design of two prototypes utilizing wavelength shifters and light guides are presented, and aspects of the research and development program are discussed.

38th International Conference on High Energy Physics

3-10 August 2016

Chicago, USA

\footnotetext{
* Speaker.

${ }^{\dagger}$ For the DUNE Collaboration

‡FERMILAB-CONF-16-604-ND-PPD
} 


\section{Introduction}

The upcoming Deep Underground Neutrino Experiment (DUNE) will study neutrino oscillation physics with an intense beam of neutrinos from Fermilab. A detector near the beam's origin will study neutrino interaction physics and enable oscillation analyses to be conducted with data taken at a far detector underground (4300 mwe) in Lead, South Dakota, 1300 km from Fermilab. This far detector will comprise four 10kt fiducial mass liquid argon (LAr) time-projection chambers (TPCs). Such a large, sensitive detector mass located deep underground will also enable many important non-beam physics measurements, including atmospheric neutrinos, neutrinos from potential supernovae, and searches for nucleon decay.

The DUNE design for single-phase LAr modules, as shown in Figure 1, contains several TPC volumes with both the anodes and cathodes submerged in LAr. Ionization electrons produced by the propagation of charged particles in LAr drift in a horizontal field to wire planes wrapped around anode plane assemblies (APAs, in red in Figure 1). TPCs are designed for highresolution tracking and energy measurements. However, to precisely determine the location of an event in the drift direction, the event time $\left(t_{0}\right)$ must be known. Since LAr is also a high-yield scintillator, the prompt signal from scintillation photons can provide $t_{0}$, yield-

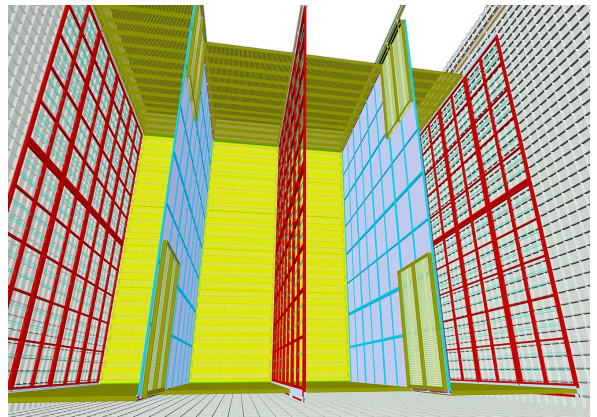

Figure 1: Depiction of a DUNE singlephase module [1]. ing $\sim \mathrm{mm}$ spatial resolution in the drift direction [1]

\section{Photon Detection System Design}

Though it is an important signal, detecting the $128 \mathrm{~nm}$ (vacuum ultraviolet, or VUV) liquid argon scintillation signal is difficult, especially in such large TPC volumes. Wavelength shifters (WLS) such as tetraphenyl butadiene (TPB) are used to convert scintillation photons into a visible signal for which detector options are more readily available. Further, a photon detection (PD) system must have significant coverage for detection of photons throughout the LAr volume while not degrading TPC performance. The LAr volume on each side of some APAs is an active TPC region (see Figure 1). For its PD system, the DUNE single-phase baseline solution is to embed light guides within the APAs, in the space between TPC wire planes, where objects $\sim 2 \mathrm{~m}$ in length and $6 \mathrm{~mm}$ in thickness can be placed. Where both neighboring regions constitute LAr volumes, an event occurring in either volume will be detected with the same light guide. A combination of WLS and light guides provides a system that converts the $128 \mathrm{~nm}$ scintillation photons to visible photons and transports converted photons to an array of silicon photomultipliers (SiPMs) at the end. In fact, $12(6 \times 6) \mathrm{mm}^{2}$ SiPMs are well-matched to the light guide dimensions. Custom electronics digitize the SiPM signals. Two main technologies utilizing WLS and light guides are being explored:

1. WLS-coated plates sit in front and behind commercially-produced wavelength-shifting light guides to convert $128 \mathrm{~nm}$ light to visible light. Converted photons reaching the light guide 
undergo a second conversion to trap some photons via total internal reflection. While requiring two wavelength shifts, the processes are decoupled. Light guides are left unaltered and the plates can be independently optimized. This design is depicted on the top of Figure 2.

2. Bare acrylic light guides are dipped in a solution containing a WLS which is then embedded in the upper layers of the acrylic. Therefore, the shift from $128 \mathrm{~nm}$ to visible happens inside the light guide and some of the light is totally internally reflected to the readout. This design only requires one wavelength shift, but the conversion and light guide properties are no longer decoupled. This design is depicted on the bottom of Figure 2.

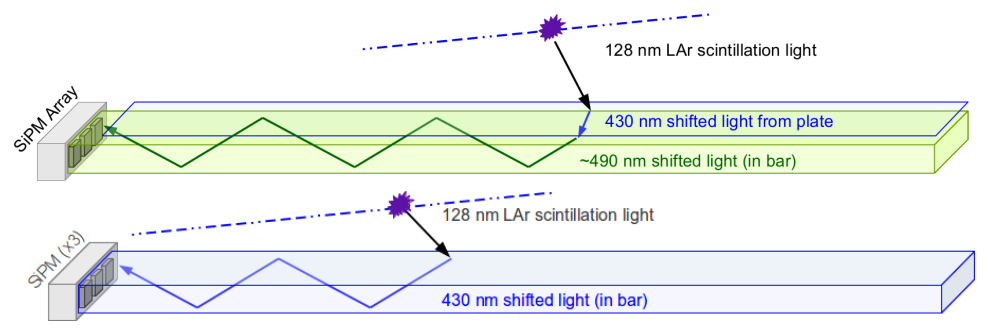

Figure 2: Depictions of PD system prototypes which utilize WLS-coated plates with light guides [2] (top) or dipped light guides [3] (bottom). While full-scale modules will have different dimensions and number of SiPMs than depicted, the illustration details the processes inolved.

\section{Testing program}

In preparation for deploying a PD system in a full-scale DUNE module, an extensive research and development program is underway. Fermilab's LAr facilities are well matched for prototype testing, with test stands capable of holding hundreds of liters of LAr. Filtered input lines and condensers maintain low-contamination volumes of LAr. Contamination monitors provide the concentrations of nitrogen, oxygen, and water to check that experimental tolerances are met. In a recent test conducted at TallBo, one such test stand, three 1 inch wide, 60 inch long dipped prototypes were tested along with one $3 \frac{3}{8}$ inch wide, 60 inch long prototype using plates and light guides. This latter prototype provided a module with the nominal width and thickness of the DUNE design. This setup allowed for side-by-side testing of the two designs.

To conduct the tests, a set of hodoscopes borrowing from the CREST cosmic ray experiment each with a two-dimensional array of 64 individual PMTs and a set of two scintillator panels each viewed by another PMT - are placed across from each other beside the dewar. This system provides a four-fold coincidence trigger to the data acquisition system on cosmic-ray muon tracks passing through the LAr volume by requring both hodoscopes detect something in PMTs from the array and adjacent scintillator paddles in coincidence. Offline analysis selects events with only one PMT in each array firing to help to reject cosmic showers and other backgrounds, and information is gained on the path taken by the muon through the LAr volume. Figure 3 contains a photo of the TallBo dewar and the hodoscopes. The setup is quite similar to that described in more detail elsewhere [3], wherein similar detector prototypes were used to study scintillation properties. Analysis of data collected from tracks through the dewar determines the characteristics of prototypes. Side- 
by-side detector comparisons can be made within the same run by comparing the signals read out. Simulations must be used to determine more fundamental properties of the PD systems.

The potential for improvement upon these designs merits further testing. For example, improved performance of the WLScoated plates will enhance the overall detection efficiency. A VUV monochromator is being used to determine the performance of WLS-coated plates at the LAr scintillation wavelength. Combining measurements of converted light from a sample plate exposed to $128 \mathrm{~nm}$ by a VUV lamp with the amount of $128 \mathrm{~nm}$ light provided by the lamp, data from the monochromator are used to measure and compare the effiencies of WLScoated plates. This testing has been done to select production methods and specific plates in an attempt to produce more efficienct pro-

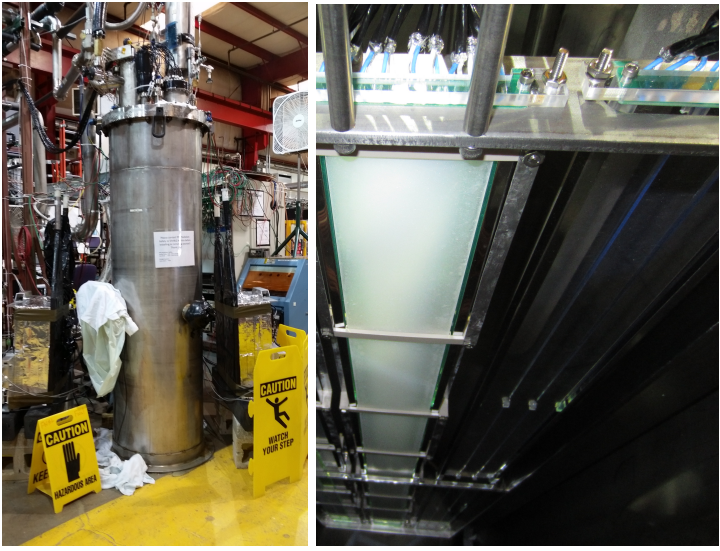

Figure 3: (Left) The TallBo test stand with hodoscopes on either side. (Right) Prototypes from the recent test. The prototype with plates and a light guide is to the left and the dipped prototypes are to the right. totypes for testing purposes. Similarly, it is a quality control test for plates to be used in producing photon dector modules of this style.

Further testing in the LAr facilities at Fermilab will continue to provide feedback on the performance of prototype PD system designs and can explore other potential PD systems which fit in a similar framework. In addition to the testing described above, PD prototypes were deployed behind wire planes in the 35-ton prototype test at Fermilab, providing system-wide operational experience. Additionally, PD system prototypes will be deployed in the protoDUNE single-phase test program at CERN, where they will be tested side-by-side along with prototype TPCs.

\section{Acknowledgments}

This work was partially supported by the Office of High Energy Physics of the DOE with grant DE-SC0010120 to Indiana University and by Brookhaven National Laboratory with grant 240296A3 to Indiana University. Fermilab is operated by Fermi Research Alliance, LLC under Contract No. DE-AC02-07CH11359 with the United States Department of Energy. The author would like to thank everyone involved in the effort of developing the DUNE photon detection system.

\section{References}

[1] R. Acciarri et al., Long-Baseline Neutrino Facility (LBNF) and Deep Underground Neutrino Experiment (DUNE) Conceptual Design Report, Volume 4 The DUNE Detectors at LBNF, arXiv:1601.02984 [ins-det].

[2] D. Whittington, Photon detection syste designs for the Deep Underground Neutrino Experiment, in proceedings of Light Detection in Noble Elements (LIDINE2015), JINST 11 (2016) C05019.

[3] D. Whittington, S. Mufson, and B. Howard, Scintillation light from cosmic-ray muons in liquid argon, JINST 11 (2016) P050016. 